\author{
Yasuhiro Matsumura* \\ Department of Internal Medicine, \\ Akishima Hospital, 1260 Nakagami-cho, \\ Akishima-shi, Tokyo, 196-0022, Japan \\ Dates: Received: 10 January, 2015; Accepted: 03 \\ March, 2015; Published: 05 March, 2015 \\ *Corresponding author: Dr. Yasuhiro Matsumura, \\ Department of Internal Medicine, Akishima Hospital, \\ 1260 Nakagami-cho, Akishima-shi, Tokyo 196-0022, \\ Japan, Tel:+81-42-546-3111; Fax: +81-42-545-5175; \\ E-mail: y-matsumura@aki-hp.jp \\ www.peertechz.com \\ ISSN: 2455-8141 \\ Keywords: Airway microbiome; \\ Airway smooth muscle; Bronchial asthma; \\ Circadian clock; Glucocorticoid; Glucocorticoid receptor; \\ Glucocorticoid resistant asthma; Non-genomic action
}

\section{Review Article \\ Recent Topics in Steroid and Asthma: Beyond the 'Classic' Concept of Action}

\begin{abstract}
Summary
Glucocorticoid (GC)s exert anti-inflammatory effects via binding to the glucocorticoid receptor (GR) (NR3C1), targeted gene expression, and protein synthesis, which need hours before the onset of the action (transactivation). GCs also suppress inflammation by direct or indirect interaction with transcription factors, such as activator protein-1 (AP-1) and nuclear factor-KB (NF-kB) (transrepression). Recently, the non-genomic actions of GCs were discovered on recognition of its rapid onset of action within seconds to minutes.

GCs target many cells and tissues, including immune and inflammatory cells, airway epithelium, and airway smooth muscle (ASM). Of these, ASM is involved in altered airway contractility. A recent study demonstrated that GCs not only suppress inflammation but also exert direct effects on ASM gene expression which influence ASM function.

GC resistance in the treatment of bronchial asthma remains a considerable clinical problem. Genes and cellular inflammatory phenotypes of glucocorticoid-resistant (GC-R) asthma have been revealed. Inflammation-associated protein kinase signaling and transcription factors affect GC actions through modulating GR function. Involvement of chromatin modifications have also been reported. Infection, reduced Vitamin D (Vit D), smoking, and obesity are preventable risk factors in GC-R asthma.

Some of these recently available results are presented in this review.
\end{abstract}

\section{Abbreviations}

AP-1: Activator Protein-1; ASM: Airway Smooth Muscle; BMAL1: Brain and Muscle ARNT Like 1; BSA: Bovine Serum Albumin; BUD: Budesonide; C/EBP: CCAAT/Enhancer Binding Protein; CEBPD: CCAAT/Enhancer Binding Brotein $\delta$; CFTR: Cystic Fibrosis Transmembrane Conductance Regulator; CLOCK: Circadian Locomotor Output Cycles Kaput; CREB: CAMP Response Element Binding Protein; CRISPLD2: Cysteine-Rich Secretory Protein LCCL Domain-Containing 2; Cry: Cryptochrone; CXCL5: Chemokine (C-X-C motif) Ligand 5; DDIT4: DNA-Damage Inducible Transcript 4; DEX: Dexamethasone; DUSP1: Dual-Specificity Phosphatase 1; FAM129A: Family with Sequence Similarity, 129 Member A; FP: Fluticasone Propionate; GATA-3: GATA Binding Protein 3; GC: Glucocorticoid; GILZ: Glucocorticoid-Induced Leucine Zipper; GR: Glucocorticoid Receptor; GRE: GC Response Element; HAT: Histone Acetyl Transferase; HDAC: Histone Deacetylase; IRF 3: IFN Regulatory Factor 3; INF: Interferon; JNK: c-JUN N-terminal Kinase; Klf15: Kruppel-Like Factor 15; LEPREL1: Leprecan-Like 1; LPS: Lipopolysaccharide; MAPK:Mitogen-Activated Protein Kinase; MKP-1: Mitogen-Activated Protein Kinase Phosphatase 1; NF-AT: Nuclear Factor of Activated T-cells; NF- $\kappa$ B: Nuclear Factor- $\kappa B$; nGRE: Negative Glucocorticoid Response Element; PBMC: Peripheral Blood Mononuclear Cell; Per: Period; RPTOR: Regulatory-Associated Protein of Mtor; S211: Serine 211; STAT 6: Signal Transducer and Activator of Transcription 6; SYNPO2: Synaptopodin 2; T-bet: T-box Transcription Factor; TCR: T-Cell Receptor; TSLP: Thymic Stromal
Lymphopoietin: VANGL1: VANGL Planar Cell Polarity Protein 1; Vit D: Vitamin D

\section{Introduction}

Several steroids were isolated from the adrenal cortex during the 1940s by Edward Kendall. GR was cloned and expressed in 1985 [1]. GCs can have an anti-inflammatory effect and a pro-apoptotic action for disease therapy. Administration of adrenal extracts was first reported to reduce the frequency of exacerbationsof asthma in 1900 [2]. Clinical trials of inhaled steroid in asthma were started in about $1970[3,4]$. Now, there is widespread use of GCs in patients with bronchial asthma, via oral, intravenous, and especially inhalation routes. However, the complete picture of GC and GR function remains to be elucidated.

The actions of GCs are a fast-moving and exciting research field. In this review, recent advances (over the last 2 to 3 years) in understanding the action and molecular mechanisms of GCs that are believed to promote anti-inflammatory effects in bronchial asthma, especially, recent findings on non-genomic effects of GCs, the mechanism by which GCs suppress inflammation and improve function in ASM, and GC resistance in bronchial asthma are summarized and discussed.

\section{Mechanisms of action of GC}

GCs exert pro and anti-inflammatory effects by both gene induction and repression. GCs act by binding to GR. Activated GRs 
can drive gene transcription via binding onto GC response elements (GREs) located in the promoter proximal region of the target gene. A number of genes that exhibit anti-inflammatory actions, including glucocorticoid-induced leucine zipper (GILZ), dualspecificity phosphatase 1 (DUSP1), and mitogen-activated protein kinase phosphatase (MKP)-1, are upregulated via a transactivation mechanism. In many GR-binding sites located far from the promoter proximal region of the target genes, binding of GR to chromatin may occur by tethering to other transcription factors [5]. GCs transcriptionally repress thymic stromal lymphopoietin (TSLP) through direct binding of GR to negative glucocorticoid response element (nGRE) [6]. GR also can actively repress target gene transcription by recruiting corepressors [7].

Nuclear GRs interact directly or indirectly with coactivator molecules. Switching off inflammatory genes through interactions with transcription factors, such as $\mathrm{AP}-1, \mathrm{NF}-\mathrm{\kappa B}$, cAMP response element binding protein (CREB), nuclear factor of activated T-cells (NF-AT), signal transducer and activator of transcription (STAT) 6 , interferon (IFN) regulatory factor (IRF) 3, STAT3, GATA binding protein 3 (GATA-3), and T-box transcription factor (T-bet), may be the major effect of GCs.

GCs act through a combination of direct inhibition of histone acetyltransferase (HAT) activity and recruitment of histone deacetylase (HDAC) to the activated transcriptional complex. Low concentrations of GCs can switch off inflammatory genes through the recruitment of HDAC2. HDAC2 acts by deacetylating GR, thereby enabling $\mathrm{p} 65-\mathrm{NF}-\mathrm{\kappa B}$ association and attenuation of proinflammatory gene transcription [8].

Recent interest in the mechanism of action of GCs is their nongenomic action. A latency of several hours is required before the onset of the genomic effects of GCs in the complex process, including ligand-receptor binding, gene expression, and protein synthesis. GCs also induce rapid non-genomic responses in seconds to minutes. The non-genomic effects of GC in asthma are becoming clearer. A physical and functional interaction between the GR and the T-cell receptor (TCR) complex [9] and membrane-bound GR induced phosphorylation of p38 mitogen-activated protein kinase (MAPK) due to DEX/bovine serum albumin (BSA) treatment [10] has been reported. Low concentrations of DEX rapidly regulate intracellular $\mathrm{pH}, \mathrm{Ca}^{2+}$ and cAMP-dependent protein kinase activity, and inhibit $\mathrm{Cl}^{-}$secretion in bronchial epithelial cells via nongenomic mechanisms [11]. A recent study showed that inhaled fluticasone propionate (FP) and budesonide (BUD) stimulate cystic fibrosis transmembrane conductance regulator (CFTR)-mediated anion transport through adenylate cyclase-mediated mechanisms in a nongenomic fashion [12]. In addition to their anti-inflammatory properties, GCs attenuate ASM contractility through non-genomic effects. It is reported that BUD can rapidly inhibit histamine-induced contractions of airway smooth muscle through a process mediated by non-genomic mechanisms [13].

\section{Effects of GC on ASM}

Although the bronchial epithelium may be a direct target for GC therapy in inflammatory airway diseases, GCs also offer clinical improvement in airway function.
GCs modulate contraction of ASM by suppressing increase in intracellular calcium level or by controlling receptors, such as muscarinic M2 or M3, histamine H1 receptors. GCs relax ASM by increasing expression of $\beta 2$-adrenoceptors in a cyclic AMPdependent manner and increase $\mathrm{Na}+\mathrm{K}+$ electrogenic pump activity in a cyclic AMP-independent manner [14].

Accumulating study results suggest that GCs not only exert effects on airway epithelial gene expression in asthma [15], but also exert effects on ASM gene expression which influence ASM function $[16,17]$. The transcriptomic profile of ASM, such as regulatoryassociated protein of mTOR (RPTOR), VANGL planar cell polarity protein 1 (VANGL1), family with sequence similarity 129 , member A (FAM129A), and leprecan-like 1 (LEPREL1), has been reported to be associated with airway hyper-responsiveness [18]. The transcriptomic profile of FAM129A and synaptopodin 2 (SYNPO2) of the airway smooth muscle layer in asthma was changed by oral prednisolone, associated with an improvement in airway hyperresponsiveness [19]. The effect of a GC on ASM associated with cysteine-rich secretory protein LCCL domain-containing 2 (CRISPLD2) has been reported, which may be a candidate gene for pharmacogenetics in asthma that regulates the anti-inflammatory effects of GCs in ASM [20]. The transcription factor Kruppel-like factor 15 (Klf15) has been reported as a GR target gene that modulates airway contractility, possibly through regulating apoptosis and proliferation of ASM [21].

ASM cells of asthmatic patients have the potential to proliferate faster than cells from control subjects under defined conditions [22]. CCAAT/enhancer binding protein (C/EBP)s are pleiotropic proteins involved in inflammation, cell differentiation and tissue remodelling [23]. ASM cells from asthmatics are deficient in C/EBP- $\alpha$, resulting in poor inhibition of smooth muscle proliferation in vitro [24]. BUD plus formoterol simultaneously activates GR and C/EBP- $\alpha$, resulting in synergistic stimulatory effects on $\mathrm{p} 21$ promoter activity and additive inhibitory effects on proliferation [25].

Thus, GCs exert direct effects on ASM by inhibiting its contractility, increasing its relaxation, inhibiting cell proliferation, and preventing the release of proinflammatory cytokines and chemokines.

\section{Molecular clock mechanism: Interaction of GC with clock components}

The GC hormone system interacts with the circadian clock, which is an endogenous biological timing mechanism. The central pacemaker is the hypothalamus, which coordinates the activities of target organs.

GR function is determined by physical interactions with clock components $[26,27]$. Recently, molecular clock mechanisms in the lung have been recognized. A heterodimer of circadian locomotor output cycles kaput (CLOCK) and brain and muscle ARNT like 1 (BMAL1) activates transcription via E-box enhancer element, resulting in Period (Per1-3) and Cryptochrone (Cry1,2) gene translation. PERs and CRYs inhibit CLOCK-BMAL1-mediated transcription. This consists of transcription-transcription feedback loops [28]. CLOCK/BMAL1, the core circadian clock components, reduce maximal GR transactivation as well as efficacy [29]. 
The therapeutic effects of dexamethasone (DEX) depend on intact clock function in the airway. Genetic ablation of the clock gene Bmal1 in bronchiolar cells disrupts rhythmic chemokine (C-X-C motif) ligand 5 (CXCL5) expression, resulting in exaggerated inflammatory responses to lipopolysaccharide (LPS) and an impaired host response to Streptococcus pneumoniae infection [30]. Thus, an epithelial circadian clock is important in controlling pulmonary inflammation and GC action.

\section{Homeostatic enhancement of GC action}

Regarding the homeostatic enhancement of the response to GC, interesting studies has been reported. Inflammatory processes may influence the response of bronchial epithelium to GC via cytokines [31]. Furthermore, pro-asthmatic cytokine-driven, MAPK-mediated, non-ligand-dependent GR activation that confers heightened GC ligand-stimulated GR signaling in ASM, suggesting proinflammatory cytokine-induced ligand-independent GR signaling, may importantly contribute to heightening GC sensitivity in asthma [32].

\section{Mechanisms involved in GC resistance}

Asthma is a heterogeneous group of disorders demonstrating overlapping phenotypes. The response to GCs varies considerably among patients with asthma [33]. Consistent differential expression of CCAAT/enhancer binding protein $\delta(C E B P D)$ and DNA-damage inducible transcript 4 (DDIT4) in asthmatics has been reported, suggesting that GC genes, whose changes in expression characterize the response to GCs, are associated with the developmental origins of asthma and treatment response [34].

Cellular inflammatory phenotypes of GC-R asthma, such as eosinophilic or neutrophilic, have been revealed. Generally, the phenotype of airway infiltration with eosinophils shows a better clinical response to GCs compared to the phenotype with neutrophils [35,36]. Neutrophils in the airway, T helper 17 (Th17) cells and interleukin (IL)-23, an IL-12-related cytokine that is essential for survival and functional maturation of Th17 cells, may be involved in the pathogenesis of severe asthma and GC resistance [37].

GR $\beta$, a splice variant of GR (GR $\alpha$ ), is up-regulated in GC-R asthma [38]. IL-23 and IL-17A/F increased the GR $\beta / G R \alpha$ ratio in peripheral blood mononuclear cell (PBMC)s [39]. GR $\beta$ does not bind GC. The role of GR $\beta$ is not clear, but a recent study demonstrated that GR $\beta$ has a gene regulatory function, which may alter GC signaling, independent of its GRa antagonism [40].

Increased p38 MAPK activation due to inflammatory stimulation and impaired MKP-1 inducibility has been observed in GC-R asthma. Activation of p38 MAPK modulates GR function, possibly as a result of phosphorylation of GR. The phosphorylation of GR at serine 211 (S211) induces GR-mediated transactivation, whereas S266 decreases GR transcriptional activities [41,42]. P38 MAPK acts negatively on GR function in ASM cells and S203 residues driving GR function as phosphorylation sites in the absence or presence of GCs [43]. A p38MAPK inhibitor (SB203580) restored GC sensitivity in PBMC from severe asthmatics, characterized by increased ex-vivo GC insensitivity, decreased GR nuclear translocation and clinically by a tendency for reduced lung function and higher use of oral GCs[44].
GC resistance in asthma may be attributed to an increase in the expression of pro-inflammatory transcription factors, such as NF- $\kappa \mathrm{B}$. Decreased GR expression with impaired nuclear translocation and subsequent inability to suppress p65 recruitment to gene promoters underlie the defective GC suppression of NF- $\mathrm{kB}$-mediated chemokine expression in ASM of severe asthma [45].

Viral infections have been identified as the most frequent triggers of asthma exacerbations. Rhinovirus infection of the airway epithelium induces GC resistance, and this process requires activation of the NF- $\mathrm{KB}$ and c-Jun N-terminal kinase (JNK) pathways [46].

In asthmatics, a reduced Vit D level might be associated with reduced GC response. Vit D inhibits p38 MAPK activation and IL-6 production and stimulates MKP-1 expression, demonstrating antiinflammatory effects in human monocytes. However, Vit D does not affect GR phosphorylation at S211 [47]. Patients with severe asthma exacerbation with Vit D deficiency showed oxidative stress and DNA damage in peripheral blood monocytes [48].

The airway microbiome potentially influences the presentation of asthma, since the airway epithelium exhibits important immunologic responses to microbial exposure. The existence of disordered microbial communities in the asthmatic airways has been highlighted [49]. Airway microbiome composition and diversity correlate with bronchial hyperresponsiveness [50]. Microbial expansion reduces cellular responses to GCs and influences the efficacy of GC treatment [51]. Gram-negative proteobacteria might be a source of airway endotoxin [52]. The association between higher sputum endotoxin level and an impaired lung function response to oral GCs, particularly in asthmatics who were never smokers, suggests that airway endotoxin might contribute to GC insensitivity in asthmatic patients [53].

Treatment not only increases but also decreases GR-dependent transcription. Expression of the GC-induced genes, GILZ and FKBP51, is up-regulated in the airways of allergen-challenged asthmatic subjects receiving inhaled BUD. GILZ reduces NF- $\mathrm{kB}-$ and AP-1-dependent transcription and IL-8 expression, resulting in an anti-inflammatory effect. On the other hand, FKBP51 may act negatively on GR activity. The induction of FKBP51 by GC may reduce therapeutic GC responsiveness. This should be taken into account when considering GC efficacy [54].

Taken together, these studies give us a greater understanding of the mechanisms of GC resistance in asthma, and this knowledge may lead us to develop new therapeutic drugs, including new GR agonists and GC-sparing therapies, in the future.

\section{Conclusions}

GCs are currently the mainstay for controlling asthma symptoms. There is a need to enhance the anti-inflammatory potential of GCs while minimizing their adverse effects. New therapies to modulate the GC response in patients with a poor response to GCs are needed to improve asthma control. From this point of view, it is important to investigate how the structure of gene promoters, GR number, the complement and abundance of co-factors, epigenetic modifications, 
and the affinity and intrinsic efficacy of GR agonists of interest influence the action of GCs $[55,56]$.

\section{References}

1. Hollenberg SM, Weinberger C, Ong ES, Cerelli G, Oro A, et al. (1985) Primary structure and expression of a functional human glucocorticoid receptor cDNA. Nature 318: 635-641.

2. Solis-Cohen (1900) The use of adrenal substance in the treatment of asthma JAMA 43: 1164-1166.

3. Brown HM, Storey G, George WH (1972) Beclomethasone dipropionate: a new steroid aerosol for the treatment of allergic asthma. Br Med J 1: 585-590.

4. Barnes PJ (2014) Glucocorticoids. Chem Immunol Allergy 100: 311-316.

5. Reddy TE, Pauli F, Sprouse RO, Neff NF, Newberry KM, et al. (2009) Genomic determination of the glucocorticoid response reveals unexpected mechanisms of gene regulation. Genome Res 19: 2163-2171.

6. Hudson WH, Youn C, Ortlund EA (2013) The structural basis of direct glucocorticoid-mediated transrepression 20: 53-58.

7. Surjit M, Ganti KP, Mukherji A, Ye T, Hua G, et al. (2011) Widespread negative response elements mediate direct repression by agonist-liganded glucocorticoid receptor. Cell 145: 224-241.

8. Ito K, Yamamura S, Essilfie-Quaye S, Cosio B, Ito M, Barnes PJ, et al. (2006) Histone deacetylase 2-mediated deacetylation of the glucocorticoid receptor enables NF-kappaB suppression. J Exp Med 203: 7-13.

9. Löwenberg M, Verhaar AP, van den Brink GR, Hommes DW (2007) Glucocorticoid signaling: a nongenomic mechanism for T-cell immunosuppression. Trends Mol Med 13: 158-163.

10. Strehl C, Gaber T, Löwenberg M, Hommes DW, Verhaar AP, et al. (2011) Origin and functional activity of the membrane-bound glucocorticoid receptor. Arthritis Rheum 63: 3779-3788.

11. Urbach V, Verriere V, Grumbach Y, Bousquet J, Harvey BJ (2006) Rapid antisecretory effects of glucocorticoids in human airway epithelium. Steroids 71 : 323-328

12. Mizutani T, Morise M, Ito $Y$, Hibino $Y$, Matsuno T, et al. (2012) Nongenomic effects of fluticasone propionate and budesonide on human airway anion secretion. Am J Respir Cell Mol Biol 47: 645-651.

13. Sun HW, Miao CY, Liu L, Zhou J, Su DF, et al. (2006) Rapid inhibitory effect of glucocorticoids on airway smooth muscle contractions in guinea pigs. Steroids 71: 154-159

14. Hirst SJ, Lee TH (1998) Airway smooth muscle as a target of glucocorticoid action in the treatment of asthma. Am J Respir Crit Care Med 158: S201-S206.

15. Woodruff PG, Boushey HA, Dolganov GM, Barker CS, Yang YH, et al. (2007) Genome-wide profiling identifies epithelial cell genes associated with asthma and with treatment response to corticosteroids. Proc Natl Acad Sci U S A 104: 15858-15863.

16. Hakonarson H, Halapi E, Whelan R, Gulcher J, Stefansson K, et al. (2001) Association between IL-1beta/TNF-alpha-induced glucocorticoid-sensitive changes in multiple gene expression and altered responsiveness in airway smooth muscle. Am J Respir Cell Mol Biol 25: 761-771.

17. Misior AM, Deshpande DA, Loza MJ, Pascual RM, Hipp JD, et al. (2009) Glucocorticoid- and protein kinase A-dependent transcriptome regulation in airway smooth muscle. Am J Respir Cell Mol Biol 41: 24-39.

18. Yick CY, Zwinderman AH, Kunst PW, Grünberg K, Mauad T, et al. (2014) Gene expression profiling of laser microdissected airway smooth muscle tissue in asthma and atopy. Allergy 69: 1233-1240.

19. Yick CY, Zwinderman AH, Kunst PW, Grünberg K, Mauad T, et al. (2013) Glucocorticoid-induced changes in gene expression of airway smooth muscle in patients with asthma. Am J Respir Crit Care Med 187: 1076 -1084.

20. Himes BE, Jiang X, Wagner P, Hu R, Wang Q, et al. (2014) RNA-Seq transcriptome profiling identifies CRISPLD2 as a glucocorticoid responsive gene that modulates cytokine function in airway smooth muscle cells. PLoS One 9: e99625.

21. Masuno K, Haldar SM, Jeyaraj D, Mailloux CM, Huang X, et al. (2011) Expression profiling identifies KIf15 as a glucocorticoid target that regulates airway hyperresponsiveness. Am J Respir Cell Mol Biol 45: 642-649.

22. Johnson PR, Roth M, Tamm M, Hughes M, Ge Q, et al. (2001) Airway smooth muscle cell proliferation is increased in asthma. Am J Respir Crit Care Med 164: 474-477.

23. Miglino N, Roth M, Tamm M, Borger P (2012) Asthma and COPD - The C/ EBP Connection. Open Respir Med J 6: 1-13.

24. Roth M, Black JL (2009) An imbalance in C/EBPs and increased mitochondrial activity in asthmatic airway smooth muscle cells: novel targets in asthma therapy? Br J Pharmacol 157: 334-341.

25. Roth M, Johnson PR, Rüdiger JJ, King GG, Ge Q, et al. (2002) Interaction between glucocorticoids and beta2 agonists on bronchial airway smooth muscle cells through synchronised cellular signalling. Lancet 360: 12931299.

26. Nader N, Chrousos GP, Kino T (2009) Circadian rhythm transcription factor CLOCK regulates the transcriptional activity of the glucocorticoid receptor by acetylating its hinge region lysine cluster: potential physiological implications. FASEB J 23: 1572-1583.

27. Lamia KA, Papp SJ, Yu RT, Barish GD, Uhlenhaut NH, et al. (2011) Cryptochromes mediate rhythmic repression of the glucocorticoid receptor. Nature 480: 552-556.

28. Dickmeis T, Weger BD, Weger M (2013) The circadian clock and glucocorticoids--interactions across many time scales. Mol Cell Endocrinol 380: 2-15.

29. Han DH, Lee YJ, Kim K, Kim CJ, Cho S (2014) Modulation of glucocorticoid receptor induction properties by core circadian clock proteins. Mol Cell Endocrinol 383: 170-180

30. Gibbs J, Ince L, Matthews L, Mei J, Bell T, et al. (2014) An epithelial circadian clock controls pulmonary inflammation and glucocorticoid action. Nat Med 20: 919-926.

31. Verheggen MM, van Hal PT, Adriaansen-Soeting PW, Goense BJ, Hoogsteden $\mathrm{HC}$, et al. (1996) Modulation of glucocorticoid receptor expression in human bronchial epithelial cell lines by IL-1 beta, TNF-alpha and LPS. Eur Respir J 9: 2036-2043.

32. Hu A, Josephson MB, Diener BL, Nino G, Xu S, et al. (2013) Pro-asthmatic cytokines regulate unliganded and ligand-dependent glucocorticoid receptor signaling in airway smooth muscle. PLoS One 8: e60452.

33. Matsumura Y (2013) Inflammatory cellular phenotypes and molecular mechanisms of glucocorticoid resistance in patients with bronchial asthma. Antiinflamm Antiallergy Agents Med Chem 12: 189-200.

34. Sharma S, Kho AT, Chhabra D, Qiu W, Gaedigk R, et al. (2014) Glucocorticoid genes and the developmental origins of asthma susceptibility and treatment response. Am J Respir Cell Mol Biol [Epub ahead of print]

35. Green RH, Brightling CE, Woltmann G, Parker D, Wardlaw AJ, et al. (2002) Analysis of induced sputum in adults with asthma: identification of subgroup with isolated sputum neutrophilia and poor response to inhaled corticosteroids. Thorax 57: 875-879.

36. Jayaram L, Pizzichini MM, Cook RJ, Boulet LP, Lemière C, et al. (2006) Determining asthma treatment by monitoring sputum cell counts: effect on exacerbations. Eur Respir J 27: 483-494.

37. Nakajima H, Hirose K (2010) Role of IL-23 and Th17 cells in airway inflammation in asthma. Immune Netw 10: 1-4.

38. Goleva E, Li LB, Eves PT, Strand MJ, Martin RJ, et al. (2006) Increased glucocorticoid receptor beta alters steroid response in glucocorticoidinsensitive asthma. Am J Respir Crit Care Med 173: 607-616. 
39. Vazquez-Tello A, Halwani R, Hamid Q, Al-Muhsen S (2013) Glucocorticoid receptor-beta up-regulation and steroid resistance induction by IL-17 and IL23 cytokine stimulation in peripheral mononuclear cells. J Clin Immunol 33: 466-478.

40. Oakley RH, Cidlowski JA (2011) Cellular processing of the glucocorticoid receptor gene and protein: new mechanisms for generating tissue-specific actions of glucocorticoids. J Biol Chem 286: 3177-3184.

41. Szatmáry Z, Garabedian MJ, Vilcek J (2004) Inhibition of glucocorticoid receptor-mediated transcriptional activation by p38 mitogen-activated protein (MAP) kinase. J Biol Chem 279: 43708-43715.

42. Miller AL, Webb MS, Copik AJ, Wang Y, Johnson BH, et al. (2005) p38 Mitogen-activated protein kinase (MAPK) is a key mediator in glucocorticoidinduced apoptosis of lymphoid cells: correlation between p38 MAPK activation and site-specific phosphorylation of the human glucocorticoid receptor at serine 211. Mol Endocrinol 19: 1569-1583.

43. Bouazza B, Debba-Pavard M, Amrani Y, Isaacs L, O'Connell D, et al (2014) Basal p38 mitogen-activated protein kinase regulates unliganded glucocorticoid receptor function in airway smooth muscle cells. Am J Respir Cell Mol Biol 50: 301-315

44. Mercado N, Hakim A, Kobayashi Y, Meah S, Usmani OS, et al. (2012) Restoration of corticosteroid sensitivity by p38 mitogen activated protein kinase inhibition in peripheral blood mononuclear cells from severe asthma. PLoS One 7: e41582.

45. Chang PJ, Michaeloudes C, Zhu J, Shaikh N, Baker J, et al. (2015) Impaired nuclear translocation of the glucocorticoid receptor in corticosteroidinsensitive airway smooth muscle in severe asthma. Am J Respir Crit Care Med 191: 54-62

46. Papi A, Contoli M, Adcock IM, Bellettato C, Padovani A, et al. (2013) Rhinovirus infection causes steroid resistance in airway epithelium through nuclear factor ?B and c-Jun N-terminal kinase activation. J Allergy Clin Immunol 132: 1075-1085

47. Zhang Y, Leung DY, Goleva E (2014) Anti-inflammatory and corticosteroid- enhancing actions of vitamin $\mathrm{D}$ in monocytes of patients with steroid-resistant and those with steroid-sensitive asthma. J Allergy Clin Immunol 133: 1744 1752.

48. Lan N, Luo G, Yang X, Cheng Y, Zhang Y, et al. (2014) 25-Hydroxyvitamin D3-deficiency enhances oxidative stress and corticosteroid resistance in severe asthma exacerbation. PLoS One 9: e111599.

49. Hilty M, Burke C, Pedro H, Cardenas P, Bush A, et al. (2010) Disordered microbial communities in asthmatic airways. PLoS One 5: e8578.

50. Huang YJ, Nelson CE, Brodie EL, Desantis TZ, Baek MS, et al. (2011) National Heart, Lung, and Blood Institute's Asthma Clinical Research Network. Airway microbiota and bronchial hyperresponsiveness in patients with suboptimally controlled asthma. J Allergy Clin Immunol 127: 372-381.

51. Goleva E, Jackson LP, Harris JK, Robertson CE, Sutherland ER, et al. (2013) The effects of airway microbiome on corticosteroid responsiveness in asthma. Am J Respir Crit Care Med 188: 1193-1201.

52. Marri PR, Stern DA, Wright AL, Billheimer D, Martinez FD (2013) Asthmaassociated differences in microbial composition of induced sputum. J Allergy Clin Immunol 131: 346-352.

53. McSharry C, Spears M, Chaudhuri R, Cameron EJ, Husi H, et al. (2014) Increased sputum endotoxin levels are associated with an impaired lung function response to oral steroids in asthmatic patients. J Allergy Clin Immunol 134:1068-1075.

54. Kelly MM, King EM, Rider CF, Gwozd C, Holden NS, et al. (2012) Corticosteroid-induced gene expression in allergen-challenged asthmatic subjects taking inhaled budesonide. Br J Pharmacol 165: 1737-1747.

55. Newton R, Leigh R, Giembycz MA (2010) Pharmacological strategies for improving the efficacy and therapeutic ratio of glucocorticoids in inflammatory lung diseases. Pharmacol Ther 125: 286-327.

56. Joshi T, Johnson M, Newton R, Giembycz MA (2015) An analysis of glucocorticoid receptor-mediated gene expression in BEAS-2B human airway epithelial cells identifies distinct, ligand-directed, transcription profiles with implications for asthma therapeutics. Br J Pharmacol 172: 1360-1378.

Copyright: (c) 2015 Matsumura Y. This is an open-access article distributed under the terms of the Creative Commons Attribution License, which permits unrestricted use, distribution, and reproduction in any medium, provided the original author and source are credited. 